\title{
Umgang mit Leistungssperren: Erfahrungsberichte gesucht!
}

\section{Michelle Salathé}

lic. Iur. Michelle Salathé, MAE, Leiterin Ressort Ethik, stv. Generalsekretärin SAMW

Der Fall eines HIV-Patienten aus dem Kanton Graubünden, der als säumiger Prämienzahler keine Medikamente erhalten hatte und Ende 2017 an den Folgen einer Begleiterkrankung starb, hat mediales Aufsehen erregt. Auch die Zentrale Ethikkommission der SAMW (ZEK) wurde aufgefordert, sich in einer Stellungnahme zur Thematik der Leistungssperren zu äussern. Um einen Einblick in die Praxis zu erhalten, führte sie 2018 eine nicht-repräsentative Umfrage durch. Um das Thema zu vertiefen, sind Erfahrungsberichte erwünscht.

Vgl. Art. 64a Abs 7 KVG: «Die Kantone können versicherte Personen, die ihrer Prämienpflicht trotz Betreibung nicht nachkommen, auf einer Liste erfassen, welche nur den Leistungserbringern der Gemeinde und dem Kanton zugänglich ist. Die Versicherer schieben für diese Versicherten auf Meldung des Kantons die Übernahme der Kosten für Leistungen mit Ausnahme der Notfallbehandlungen auf und erstatten der zuständigen kantonalen Behörde Meldung über den Leistungsaufschub und dessen Aufhebung nach Begleichung der ausstehenden Forderungen.» Vgl. NZZ vom 13.7.2018 "Schwarze Listen kommen auf die Abschussliste».
Seit 2012 sind die Kantone verpflichtet, 85 Prozent der nicht bezahlten Prämien und Kostenbeteiligungen zu übernehmen, für die ein Verlustschein vorliegt. ${ }^{1} \mathrm{Zu}$ ihrer Entlastung können sie eine «schwarze Liste» von säumigen Prämienzahlern führen. In der Folge gilt für diese Patientinnen und Patienten eine Leistungssperre, und es sind nur noch Notfallbehandlungen gewährleistet. Diese «schwarzen Listen» werden kontrovers diskutiert; viele Kantone haben eine solche Liste gar nicht erst eingeführt oder sind dabei, sie wieder abzuschaffen. $^{2}$

Die Zentrale Ethikkommission der SAMW (ZEK) hat das Thema aufgenommen und 2018 eine nicht-repräsentative Umfrage durchgeführt, um sich ein Stimmungsbild aus der Praxis zu verschaffen. Befragt wurden Chefärztinnen und Chefärzte von Notfallstationen der fünf Universitätsspitäler und von acht ausgewählten Kantonsspitälern. Im Vordergrund stand die Frage, ob die aktuelle Praxis mit der ärztlichen Behandlungs- und Fürsorgepflicht vereinbar sei. Zehn Chefärzte (drei Universitätsspitäler und sieben Kantonsspitäler) retournierten den Hauptfragebogen zum stationären Bereich, die Zusatzfragen zum ambulanten Bereich beantworteten sechs Chefärzte.

Von den zehn antwortenden Ärztinnen und Ärzten gaben sechs an (zwei aus Universitätsspitälern, vier aus Kantonsspitälern), dass in ihrem Kanton eine solche «schwarze Liste» geführt wird. Eine Mehrheit dieser Spitäler verfügt auch über eine generelle Weisung zum Umgang mit Patienten mit unklarer oder unvollstän- diger Kostendeckung. Allerdings berichtete nur einer von zehn Chefärzten (Universitätsspital), dass tatsächlich Leistungssperren ausgesprochen werden. In diesem Universitätsspital wird ein entsprechender Vermerk im Krankendossier durch die Patientenadministration, die Abteilung Kostensicherung oder den behandelnden Arzt eingefügt. Ein solcher Vermerk hat jedoch bei unaufschiebbarer Notfallversorgung (inkl. Notfallbehandlungen im Sinne einer unmittelbar und zwingend erforderlichen Langzeitbehandlung) keine Leistungseinschränkung zur Folge. Eingeschränkt werden medizinische Behandlungen, die mittelbar durchgeführt werden können; als Beispiele werden Medikamenteneinstellung und Schwangerschaftsuntersuchung genannt.

\section{Ist die aktuelle Praxis mit der Behandlungs- und Fürsorgepflicht vereinbar?}

Explizit wurde die Frage gestellt, ob die Ärztinnen und Ärzte persönlich Situationen erlebt hätten, die mit ihrer Behandlungs- und Fürsorgepflicht nicht vereinbar gewesen seien. Niemand gab an, eine solche Situation erlebt zu haben. Der Chefarzt eines Universitätsspitals hielt fest: "Wir gehen als Notfallärzte sehr weit in der Abklärung/Therapie von nicht-versicherten Patienten. Natürlich kann dies zu Konflikten mit der Administration führen, aber für uns geht die sinnvolle Behandlung vor.» Ein Thema sei jedoch die Unterbelegung der Notfallstationen, die Lastenverteilung zwischen öf- 
fentlichem und privatem Spital oder der Hausarzt, der Patienten abweise.

\section{Unterschiede zwischen ambulant und stationär?}

Aufgrund der Antworten kann vermutet werden, dass in den öffentlichen Spitälern alle Patientinnen und Patienten den Zugang zu unaufschiebbarer Notfallversorgung erhalten und darunter auch Notfallbehandlungen im Sinne einer unmittelbar zwingend erforderlichen Langzeitbehandlung fallen. Vor diesem Hintergrund erstaunt es, dass dennoch sieben Befragte angaben, dass sie es sinnvoll fänden, wenn sich die ZEK der SAMW in einer Stellungnahme zur Thematik der Leistungssperren äussern würde. Eine Erklärung für diesen Wunsch könnten die Antworten aus der Zusatzbefragung zum ambulanten Bereich geben.

\section{«Im öffentlichen Spital erhalten Patienten mit Leisstungssperren die notwendige Behandlung.»}

In Bezug auf die zwingend erforderliche ambulante Weiterbehandlung (z.B. onkologische Therapie) gab die Hälfte der Antwortenden an, dass an ihrem Spital Medikamente nicht direkt abgegeben werden, sondern ein Rezept ausgestellt wird. Der Chefarzt eines Kantonsspitals formulierte dies so: «Im öffentlichen Spital sehe ich kein relevantes Problem. Die Patienten mit Leistungssperren erhalten die notwendige Behandlung. Problematisch ist, wenn die Apotheke keine Medikamente mehr abgibt (Beispiel erlebt: herztransplantierter Patient, der die Prämien nicht mehr bezahlt hat).» Auch wenn die Ergebnisse dieser nicht-repräsentativen Umfrage nicht alarmierend sind, ist es wichtig, das
Thema der Leistungssperren im Auge zu behalten. Es besteht kein Grund zur Annahme, dass die Anzahl säumiger Prämienzahlerinnen und -zahler abnimmt. Gleichzeitig steigt der Druck auf die Spitäler, wirtschaftlich zu sein. Dies könnte zu Strategien führen,

\section{Der Druck auf die Spitäler, wirtschaftlich zu sein, steigt.}

Patientinnen und Patienten vermehrt «abzuwimmeln», die Kosten verursachen, auf denen das Spital «sitzenbleibt».

\section{Erfahrungsberichte zur Vertiefung des Themas}

Die ZEK der SAMW ist sehr interessiert an Erfahrungsberichten zu folgenden Themen:

- Ist die Abgrenzung zwischen Notfallbehandlung und weiteren (erforderlichen) Behandlungen klar geregelt?

- Wer bestimmt in der Praxis, ob ein behandlungspflichtiger Notfall vorliegt oder nicht?

- Wer bestimmt, welche Behandlungen über den Notfall hinaus erfolgen?

- Gibt es Patientengruppen, bei denen der Zugang zur medizinischen Notfallversorgung erschwert ist?

- Gibt es Schnittstellen (z.B. stationär-ambulant), die dazu führen, dass eine zwingend erforderliche Weiterbehandlung nicht sichergestellt ist?

- Ist es erwünscht, dass sich die ZEK der SAMW zum Thema «Notfallbehandlung und Leistungssperren» äussert?

Rückmeldungen können an die aufgeführte Korrespondenzadresse geschickt werden. 